\title{
Prevalence of silent breast cancer in autopsy specimens, as studied by the disease being held by image-guided biopsies: The pilot study and literature review
}

\author{
ZACHAROULA SIDIROPOULOU ${ }^{1}$, ANA PAULA VASCONCELOS ${ }^{2}$, CRISTIANA COUCEIRO $^{2}$, \\ CARLOS DOS SANTOS ${ }^{3}$, ANA VIRGINIA ARAÚJO ${ }^{1}$, INÊS ALEGRE ${ }^{1}$, CLAUDIA SANTOS ${ }^{1}$, \\ FILIPA COSTA $^{1}$, VANESSA HENRIQUES ${ }^{4}$, CARLOS NEVES $^{1}$, FÁTIMA CARDOSO $^{5}$ and PERE GASCON ${ }^{6}$ \\ Departments of ${ }^{1}$ General Surgery and ${ }^{2}$ Radiology, Hospital São Francisco Xavier, Estrada do Forte do Alto do Duque, \\ 1449-005 Lisbon; ${ }^{3}$ Instituto Nacional de Medicina Legal e Ciências Forenses, I.P.-Delegação Sul, 1169-201 Lisbon; \\ ${ }^{4}$ Department of Pathology, Hospital São Francisco Xavier, Estrada do Forte do Alto do Duque, 1449-005 Lisbon; \\ ${ }^{5}$ Breast Unit, Centro Champalimaud, 1400-038 Lisbon, Portugal; ${ }^{6}$ Laboratory of Molecular \\ and Translational Oncology-CELLEX, 08035 Barcelona, Spain
}

Received February 22, 2017; Accepted May 10, 2017

DOI: $10.3892 /$ mco.2017.1299

\begin{abstract}
Breast cancer epidemiological patterns vary in European countries, which present different incidence rates. Data have suggested that the reduction in breast cancer mortality is not only due to the early detection of the disease, but is, in almost equal part, due to screening and to the advances that have been made in molecular medicine and the development of novel therapies. The aim of the present study is to quantify the actual number of cases of breast cancer present in both of the sexes by calculating the prevalence of silent breast cancer in corpses. To achieve this quantification, bilateral subcutaneous radical mastectomies are performed in corpses of either sex above 40 years of age that lacked any clinical manifestation of the disease, and where the breast cancer or its complications was not the cause of death. Only five publications exist in the international literature based on medico-legal autopsies that were designed to define the 'natural reservoir' of the disease. To the best of our knowledge, the present study is the first one to appraise breast tissue via imaging by means of orienting the biopsy incision. In conclusion, to the best of our knowledge, the design of the present study is the first of its type, where image-guided biopsies are used to define the prevalence of silent breast cancer. The study aims to demonstrate that the 'disease reservoir' is, in reality, higher than was originally considered to be so. Furthermore,
\end{abstract}

Correspondence to: Miss Zacharoula Sidiropoulou, Department of General Surgery, Hospital São Francisco Xavier, Estrada do Forte do Alto do Duque, 1449-005 Lisbon, Portugal

E-mail: zachsidmd@gmail.com

Key words: silent breast cancer, breast cancer prevalence, breast cancer epidemiology, cancer and forensic autopsies, breast cancer overtreatment the study aims to contribute towards an improved definition of the disease by determining which tumour profiles potentially do not benefit from aggressive treatments (for example, in case where a high prevalence of low-grade ductal carcinoma in situ is to be detected). According to our pilot study, this analysis represents a feasible protocol.

\section{Introduction}

Breast cancer epidemiological patterns (1) vary in European countries, presenting different incidence rates (49-148 new cases per 100,000 women) with a narrower, but still variable, range of mortality (15-36 new cases per 100,000 women).

In Portugal, there has been a gradual and progressive increase in the incidence of female breast cancer, and a progressive decrease in the mortality rate, which, according to the latest publisheddata, is 118.5 and 30.4 cases per 100,000 women, respectively [statistics provided by the Directorate-General of Health (Direção-General da Saúde), 2016; see https:/www.dgs.pt/ em-destaque/portugal-doencas-oncologicas-em-numeros-201511. aspx]. According to the same report, the national screening programme covers $67.70 \%$ of the target population, with a population adhesion rate of $60.89 \%$ (www.dgs.pt.).

Breast cancer incidence and mortality patterns differ significantly among different regions within Portugal. Furthermore, the capital area (Lisbon) of the country is not officially screened, and the majority of the population is followed in private or general practice settings.

Male breast cancer is a very rare disease, comprising $\sim 1 \%$ of breast cancers, and data are generally scant. One national study reported the diagnosis and treatment of 166 cases of male patients with breast cancer in Portugal between 1970 and 2013 (https://fenix.tecnico.ulisboa.pt/downloadFile/395145917396/resumo.pdf.). Portugal is a participant in the International Male Breast Cancer Program, coordinated by the European Organization for Research and Treatment of Cancer (EORTC) and run in conjunction with the Breast 
International Group (BIG) and the North American Breast Cancer Group (NABCG) networks.

Through increasing public awareness and improving screening programmes, the early detection of breast cancer has been made possible, resulting in an increase in the incidence of small breast tumours. However, the incidence of advanced metastatic breast cancer remains stable. Approximately 10-15\% of breast cancers in Portugal are diagnosed at stage IV, and almost one-third of the early breast cancers that are detected eventually relapse. Data have suggested that the reduction in breast cancer mortality is not only due to the early detection of the disease, but is, in almost equal part, a consequence of screening and the advances that have been made in terms of molecular medicine and the development of novel therapies (Clinical Science Symposium: New Insights into Epidemiology and Outcomes, ECCO, abs. no. O-410, 2014; http://ec.europa. eu/eurostat/statistics-explained/index.php/Cancer_statistics.).

The aim of the present study is to quantify the actual number of cases of breast cancer present in both sexes by calculating the prevalence of silent breast cancer in corpses. The intention is to quantify the cases of existing cancers, those not that had clinically manifested themselves. The results of our pilot study are consequently shown in the present case study. In the international literature, there are only five publications based on medico-legal autopsies that were designed to define the 'natural reservoir' of the disease. To the best of our knowledge, the present study is the first one to appraise breast tissue via imaging by means of orienting the biopsy incision.

\section{Patients and methods}

Literature review. In the present study, a thorough MEDLINE database search (from 1953 to the present day) was performed using the medical subject heading (MeSH) terms of 'breast' AND 'autopsy/ies', where, after the exclusion of case reports, hospital autopsies, breast benign disease and series over autopsies in patients with breast cancer, five publications (2-6) were identified, one of them being a meta-analysis (4) of the papers published between 1966-1997.

Table I summarises the five relevant studies that were identified. The most recent of the studies, published in 2015 by Stalsberg et al (5), did not enable an improved evaluation of the 'silent breast cancer' phenomenon, since it was not designed to characterise the disease 'reservoir' in the study population: The sample remained low, the age limits were outside the target population and the biopsy technique was neither oriented nor extensive.

Although the conclusion indicated that 'to definitively characterise the ductal carcinoma in situ (DCis) reservoir, a large prospective study of the age-specific prevalence of occult breast cancer is sorely needed' (4), and, in spite of the controversies of breast cancer screening and eventual overdiagnosis, hardly any studies have been performed since 1987, and the present review highlights the requirement for such a broad study.

Objectives. The present study aims to achieve several objectives, namely: i) to determine silent breast cancer prevalence in both genders; ii) to identify the specific profiles that influence the clinical manifestation of the disease; and iii) to characterise the age distribution of the silent breast cancer in the population

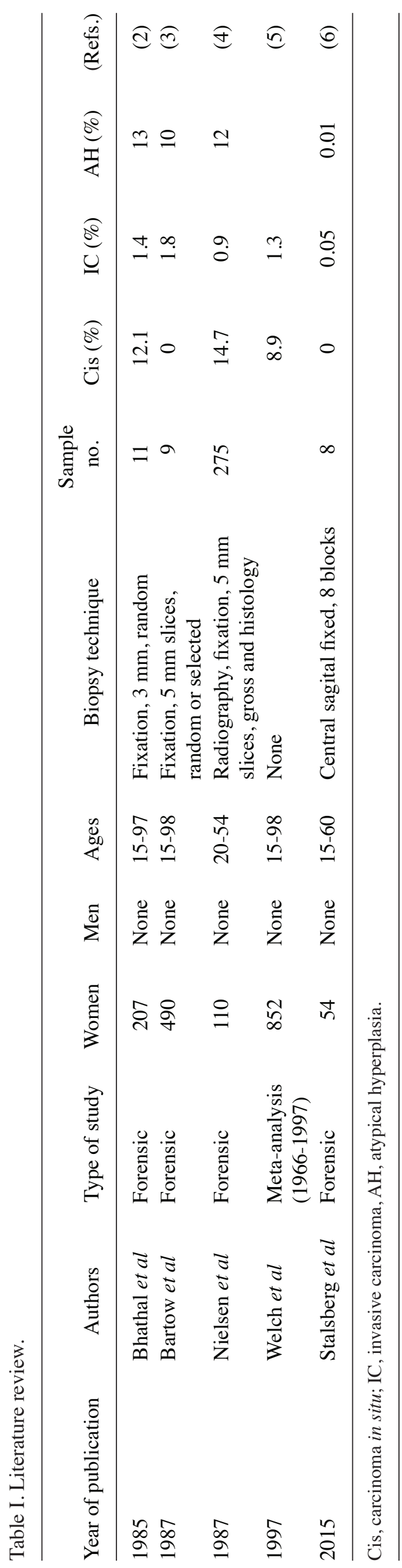


under study. The following inclusion criterion is implemented: Corpses of both sexes aged 40 or over. The exclusion criteria are mortality due to breast cancer and/or its complications, and breast tissue damage (i.e., violent death with breast injury).

Study design. Since the true prevalence of the disease has yet to be elucidated, the worst-case scenario was considered. Specifically, it was speculated that, if $50 \%$ of the studied population had undetected breast cancer, with a $5 \%$ error margin, then $384\left[\mathrm{n}=\mathrm{P} \times(100-\mathrm{P}) \times \mathrm{z}^{2} / \mathrm{d}^{2}\right.$, where $\mathrm{P}$ is the anticipated prevalence, $\mathrm{d}$ is the desired precision, and $\mathrm{z}$ is the appropriate value from the normal distribution for the desired confidence] corpses of each of the sexes would subsequently need to be examined. The samples comprising the study population were taken from the National Institute of Legal Medicine and Forensic Science (Lisbon, Portugal), following the proper tissue collection authorisation procedure.

The benefit of forensic autopsies lies in the relatively uniform age distribution of the population under study, unlike hospital samples (Table II). According to the standard procedure, once the eligibility criteria have been fulfilled and the sample collection authorisation cleared, bilateral subcutaneous modified radical mastectomy in each fresh cadaver is performed at the National Institute of Legal Medicine and Forensic Science. Tissues are subsequently transported within an appropriate container to the Hospital São Francisco Xavier (Lisbon, Portugal), and submitted for inspection, palpation, ultrasound and mammography by breast radiologists (Figs. 1-4). The imaging of the collected tissues are performed using the GE Healthcare digital mammography system, Senographe Essential $^{\mathrm{TM}}$ (GE Healthcare Bio-Sciences, Pittsburgh, PA, USA), using an X-ray beam of $27 \mathrm{kV}$ (range, 60-70 mA) and 10-15 decanewtons (daN) compression, depending on tissue density and size. The visualisation screen comprised 5 megapixels of resolution (GE Healthcare LOGIQ ${ }^{\mathrm{TM}}$ S7 Expert ultrasound system, with a medium frequency of 9-15 MHz; GE Healthcare Bio-Sciences).

Breast tissue, classified as Breast Imaging Reporting and Data System (BI-RADS) (7) category 3 or higher, is submitted to wire-guided surgical biopsy by a breast surgeon. These samples are subsequently analysed in the pathology department by an experienced hospital breast pathologist and, in case of doubt, a second pathologist, located outside the hospital, also reviews the samples.

The time frame of the study is 24-36 months, depending on the cadaver recruitment. The details of the data collection sheet are shown in Fig. 5; essentially, information concerning the patient's profile (age, ethnicity, cause of mortality, breast screening adhesion, comorbidities, medications and breast cancer risk factors), the gland's characteristics (size, weight and dimensions), the imaging characterisation of suspicious lesions (microcalcifications, stromal distortions, nodules, and so forth), lesion size, histological type, immunohistochemical profile and molecular surrogates were sought afterwards. The data collected are analysed by the hospital's statistical team.

\section{Results and discussion}

Pilot study/feasibility report. The pilot study comprised the results of the first 7 of each of the sex-specific, bilateral,
Table II. Age distribution.

Age (years) No. of corpses

30-39 74

$40-49$

$50-59$

186

60-69

127

$70-79$

149

80-89

96

$>90$

1

Forensic autopsies were performed in 2014 at the National Institute of Forensic Science (Lisbon, Portugal).

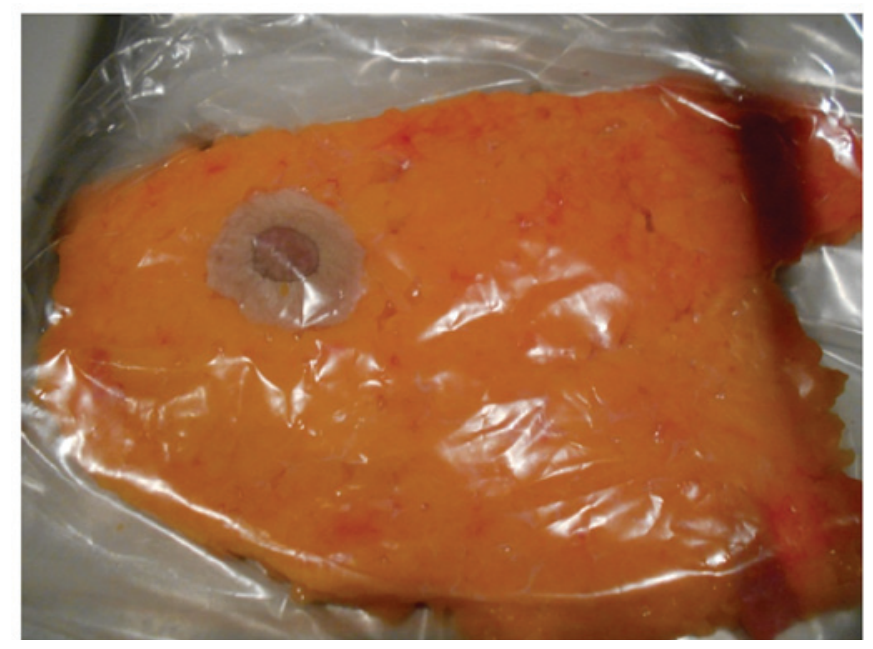

Figure 1. Left female breast sample.

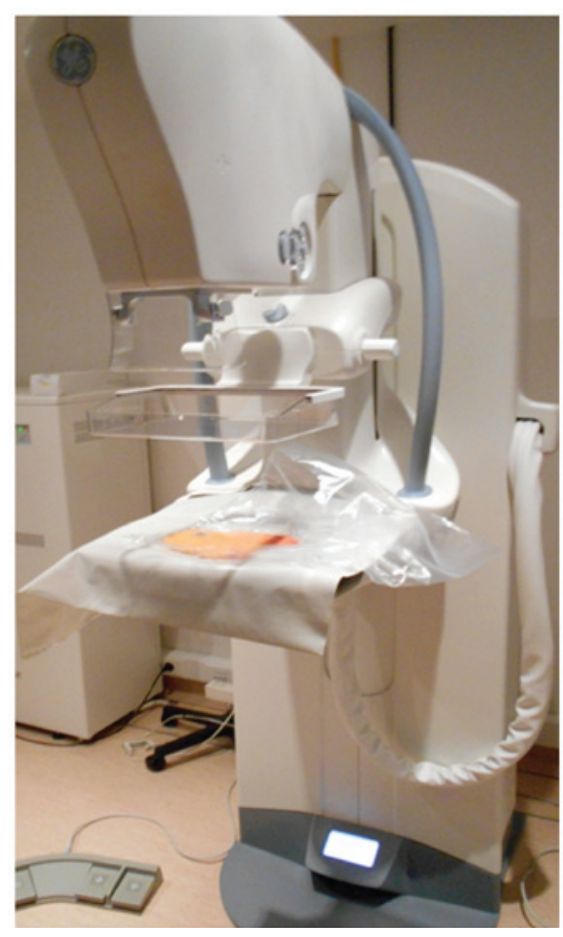

Figure 2. Mammography device and placing. 


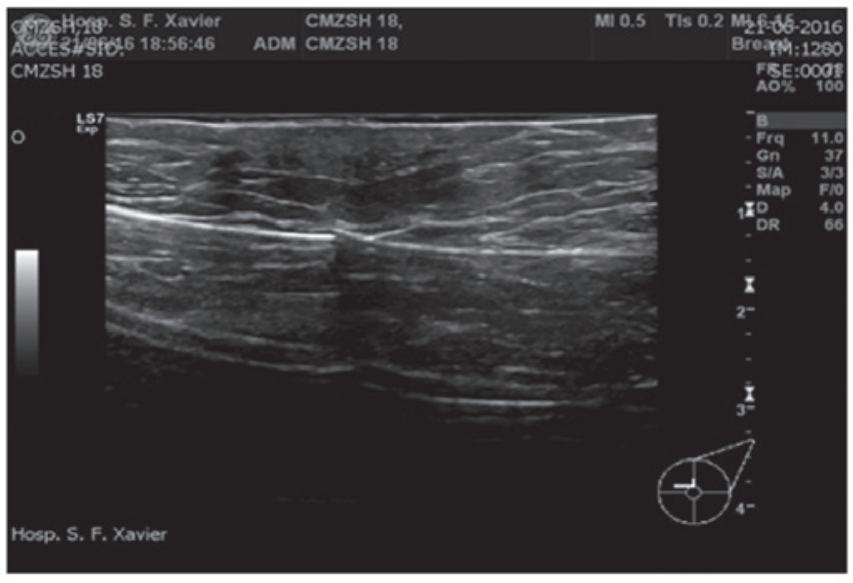

Figure 3. Ultrasonographic aspect of the tissue.

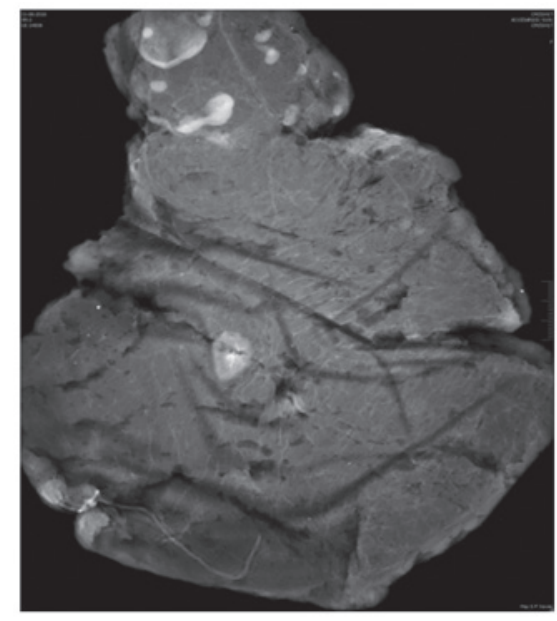

Figure 4. Mammography of the tissue.

DONOR NO

\begin{tabular}{|c|c|c|c|}
\hline \multicolumn{4}{|l|}{ Age (years) } \\
\hline Ethnicity & $\begin{array}{l}\text { Caucasian' } \\
\text { White }\end{array}$ & $\begin{array}{l}\text { Negroid/ } \\
\text { Black }\end{array}$ & $\begin{array}{l}\text { Asian/ } \\
\text { Mongoloid }\end{array}$ \\
\hline \multicolumn{4}{|l|}{ Cause of death } \\
\hline \multicolumn{4}{|l|}{ BMI } \\
\hline Breast screening & YES & NO & $\mathrm{N} / \mathrm{A}$ \\
\hline \multicolumn{4}{|l|}{ Comorbidities } \\
\hline Medication & & & \\
\hline
\end{tabular}

\begin{tabular}{|c|c|c|}
\hline GLAND & Right & Left \\
\hline \multicolumn{3}{|l|}{ Weight $(g)$} \\
\hline \multicolumn{3}{|l|}{ Size $(\mathrm{cm} \times \mathrm{cm})$} \\
\hline \multicolumn{3}{|l|}{ BI-RADS } \\
\hline \multicolumn{3}{|c|}{ Size of the lesion $(\mathrm{cm})$} \\
\hline \multicolumn{3}{|c|}{ Characteristics imaging } \\
\hline \multicolumn{3}{|c|}{ Mics } \\
\hline \multicolumn{3}{|c|}{ Nadules } \\
\hline \multicolumn{3}{|c|}{ Architectural distortion } \\
\hline \multicolumn{3}{|c|}{ Other lesions } \\
\hline \multicolumn{3}{|l|}{ Quadrant } \\
\hline Number of samples & & \\
\hline
\end{tabular}

\begin{tabular}{|c|c|c|}
\hline \multirow[t]{2}{*}{ BIOPSY } & \multirow[b]{2}{*}{ Right } & \multirow[b]{2}{*}{ Left } \\
\hline & & \\
\hline \multicolumn{3}{|c|}{$\begin{array}{l}\text { Histology (CDI, CLI, CDis, } \\
\text { CLis, ther histologies) }\end{array}$} \\
\hline \multicolumn{3}{|c|}{$\begin{array}{l}\text { Molecular surrogate } \\
\text { (luminal a, luminal b, HER2, } \\
\text { triple negative) }\end{array}$} \\
\hline \multicolumn{3}{|c|}{ RE } \\
\hline \multicolumn{3}{|l|}{$\mathbf{R P}$} \\
\hline \multicolumn{3}{|l|}{ Her2 } \\
\hline \multicolumn{3}{|l|}{ Ki 67} \\
\hline \multicolumn{3}{|l|}{ Grade } \\
\hline RA (in triple negative) & & \\
\hline
\end{tabular}

Figure 5. Details of the data collection document. BMI, body mass index; BI-RADS, breast imaging reporting and data system; Mic, microcalcification; CDI, invasive ductal carcinoma; CLI, invasive lobular carcinoma; CDis, ductal carcinoma in situ; CLis, lobular carcinoma in situ; HER2, human epidermal growth factor receptor 2; ER, estrogen receptor; PR, progesterone receptor; AR, androgen receptor.

modified radical mastectomy performed, and these details are shown in Tables III and IV for women and men, respectively.

It remains too early to present any conclusions regarding our original hypothesis, i.e., that the prevalence of silent breast cancer is going to be higher compared with the actual incidence of breast cancer. However, at the present time, it is possible to state that: (i) it is feasible to execute the prevalence definition by enlarging our time frame up to 36 months, since the 


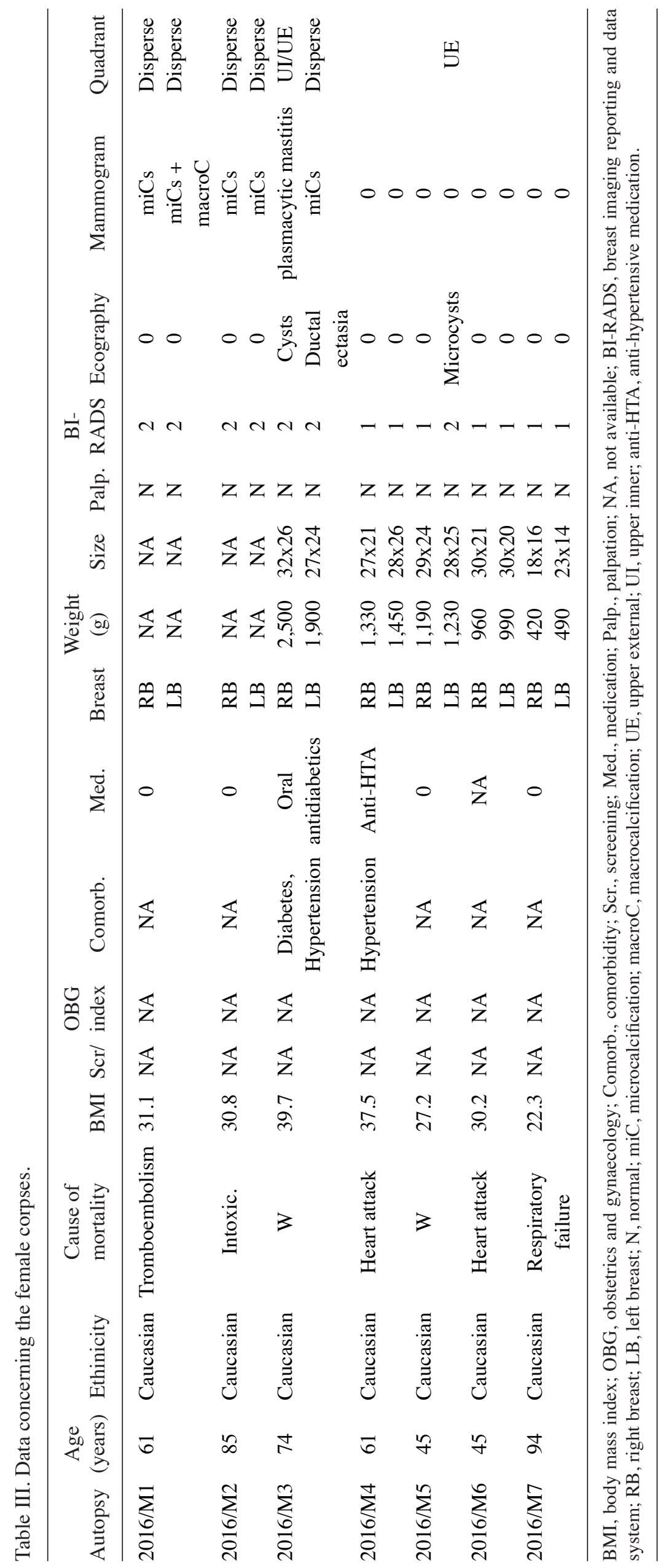




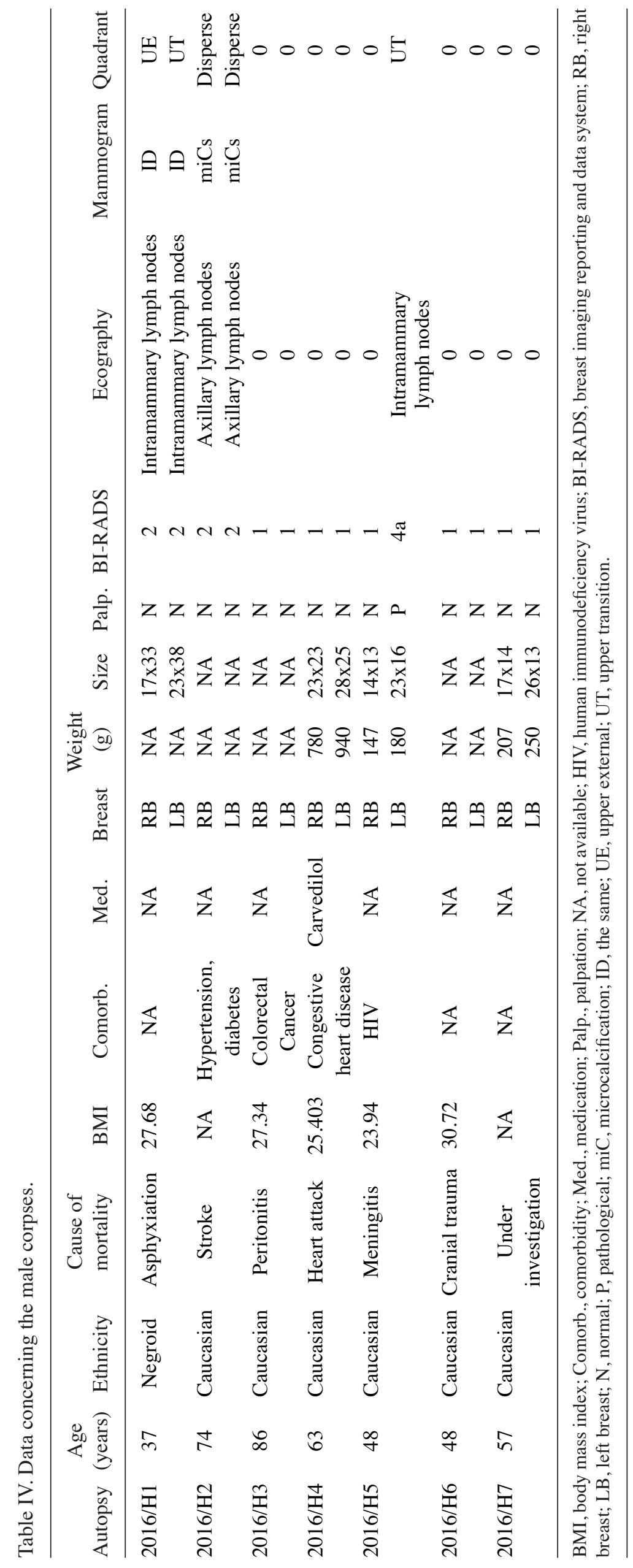


actual rate of recruitment is lower than that which was initially planned; (ii) the tissues collected in the fresh cadaver may be analyzed by means of imaging without tissue degradation, up to $48 \mathrm{~h}$ post-collection; and (iii) the corpse's specificities in terms of gynaecological/obstetric or medication and comorbidities profile was not able to be established for legal reasons (no access to personal files). For this reason, the determination of potential protective or harming factors is not possible.

In conclusion, to the best of the authors' knowledge, the design of the present study is the first of its kind, where image-guided biopsies will be used to define the silent breast cancer prevalence. The authors' aim is to contribute towards an improved understanding of the disease and its behaviour. In the case where a high prevalence of the disease is detected, this could imply indolent disease frequently identified in older age, without compromising either the quality of life or the life expectancy of the patient. In the case where the prevalence of the disease is low, it may be argued that this is due to the fact that the disease is extremely aggressive or rare, and therefore screening protocols may benefit from reviewing age boundaries or imaging and timing protocols. The present study aims to demonstrate that the disease 'reservoir' is higher than is actually considered to the case. Another objective of the study is to contribute towards an improved definition of the disease by determining which tumour profiles potentially do not benefit from aggressive treatments (for example, in case where a high prevalence of low-grade ductal carcinoma in situ is detected). According to our pilot study, this analysis represents a feasible protocol.

\section{Acknowledgements}

The authors would like to thank all the hospital staff that supported this study. The present study was supported by the
Hospital São Francisco Xavier, Centro Hospitalar de Lisboa Ocidental (CHLO), and by the Instituto Nacional de Medicina Legal Delegação do Sul, Serviço de Clínica e Patologia Forenses (Lisbon, Portugal).

\section{References}

1. Ferlay J, Steliarova-Foucher E, Lortet-Tieulent J, Rosso S, Coebergh JW, Comber H, Forman D and Bray F: Cancer incidence and mortality patterns in Europe: Estimates for 40 countries in 2012. Eur J Cancer 49: 1374-1403, 2013.

2. Bhathal PS, Brown RW, Lesueur GC and Russell IS: Frequency of benign and malignant breast lesions in 207 consecutive autopsies in Australian women. Br J Cancer 51: 271-278, 1985.

3. Bartow SA, Pathak DR, Black WC, Key CR and Teaf SR: Prevalence of benign, atypical, and malignant breast lesions in populations at different risk for breast cancer. A forensic autopsy study. Cancer 60: 2751-2760, 1987.

4. Nielsen M, Thomsen JL, Primdahl S, Dyreborg U and Andersen JA: Breast cancer and atypia among young and middle-aged women: A study of 110 medicolegal autopsies. Br J Cancer 56: 814-819, 1987.

5. Welch HG and Black WC: Using autopsy series to estimate the disease 'reservoir' for ductal carcinoma in situ of the breast: how much more breast cancer can we find? Ann Intern Med 127: 1023-1038, 1997.

6. Stalsberg H, Adjei EK and Owusu-Afriyie O: No difference in the prevalence of benign breast changes between women from Ghana and Norway: An autopsy study. Breast Cancer Res Treat 151: 177-182, 2015.

7. D'Orsi CJ, Sickles EA, Mendelson EB, Morris EA, et al: ACR BI-RADS ${ }^{\circledR}$ Atlas, Breast Imaging Reporting and Data System. Reston, VA, American College of Radiology, 2013. 